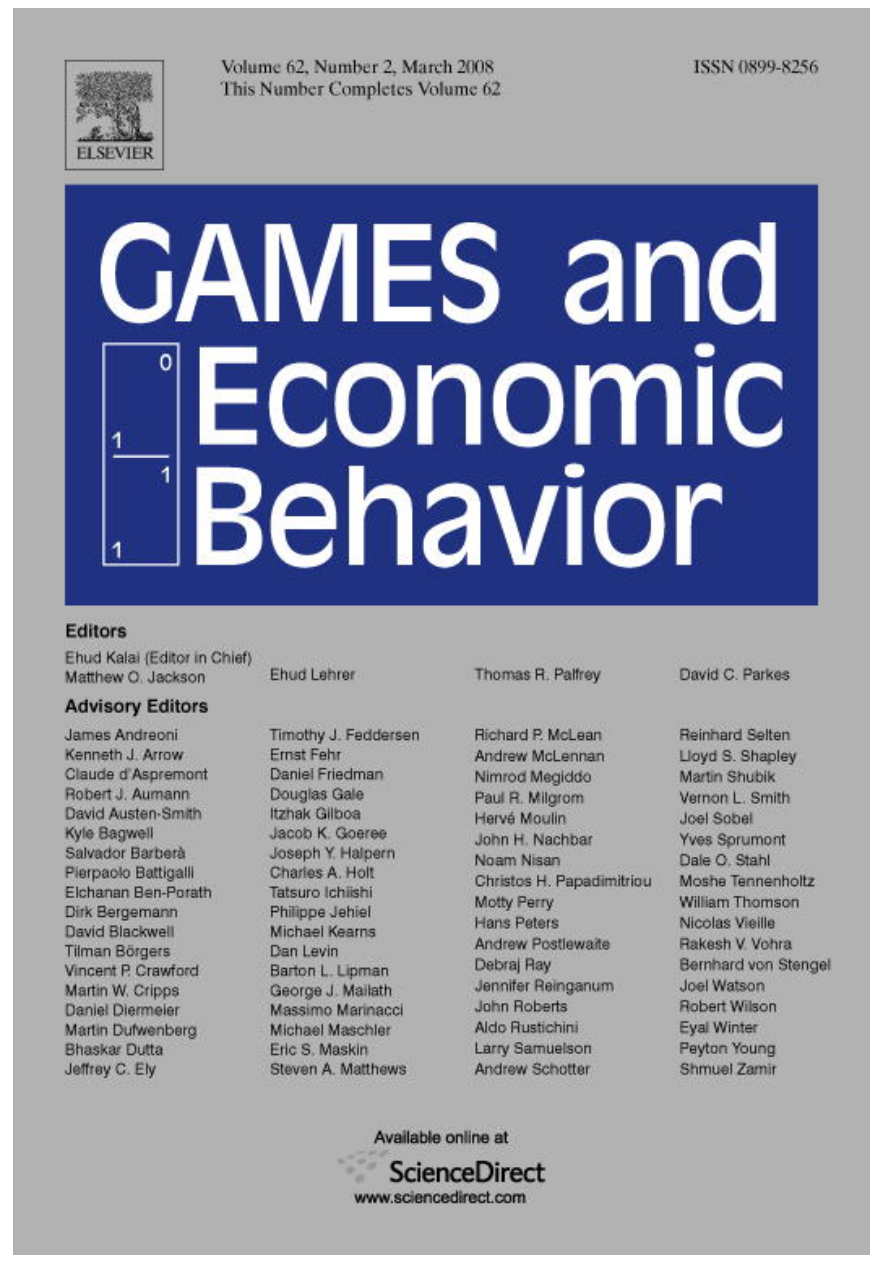

This article was published in an Elsevier journal. The attached copy

is furnished to the author for non-commercial research and education use, including for instruction at the author's institution, sharing with colleagues and providing to institution administration.

Other uses, including reproduction and distribution, or selling or licensing copies, or posting to personal, institutional or third party websites are prohibited.

In most cases authors are permitted to post their version of the article (e.g. in Word or Tex form) to their personal website or institutional repository. Authors requiring further information regarding Elsevier's archiving and manuscript policies are encouraged to visit: 


\title{
Playing the wrong game: An experimental analysis of relational complexity and strategic misrepresentation
}

\author{
Giovanna Devetag ${ }^{\mathrm{a}, *}$, Massimo Warglien ${ }^{\mathrm{b}}$ \\ a Department of Law and Management, University of Perugia, Via Pascoli 20, 06123 Perugia, Italy \\ ${ }^{\mathrm{b}}$ Department of Business Economics and Management, Ca' Foscari University of Venice, \\ San Giobbe-Cannaregio 873, Venice, Italy
}

Received 20 December 2001

Available online 22 September 2007

\begin{abstract}
It has been suggested that players often produce simplified and/or misspecified mental models of strategic decisions [Kreps, D., 1990. Game Theory and Economic Modeling. Oxford Univ. Press, Oxford]. We submit that the relational structure of players' preferences in a game is a source of cognitive complexity, and may be an important driver of such simplifications. We provide a classification of order structures in twoperson games based on the properties of monotonicity and projectivity, and present experiments in which subjects construct representations of games of different relational complexity and subsequently play the games according to these representations. Experimental results suggest that relational complexity matters. More complex games are harder to represent, and this difficulty seems correlated with short term memory capacity. In addition, most erroneous representations are simpler than the correct ones. Finally, subjects who misrepresent the games behave consistently with such representations, suggesting that in many strategic settings individuals may act optimally on the ground of simplified and mistaken premises.
\end{abstract}

(c) 2007 Elsevier Inc. All rights reserved.

JEL classification: $\mathrm{C} 70 ; \mathrm{C} 72 ; \mathrm{C} 91 ; \mathrm{D} 01$

Keywords: Pure motive; Mixed motive; Preferences; Bi-orders; Language; Cognition; Projectivity; Monotonicity;

Short-term memory; Experiments

\footnotetext{
* Corresponding author. Fax +390755855257.

E-mail addresses: devetag@unipg.it (G. Devetag), warglien@unive.it (M. Warglien).
} 


\section{Introduction}

While it is generally assumed that the structure of a game is well understood and common knowledge among players, some game theorists have challenged this assumption. For example, Kreps (1990) has suggested that "in choosing his actions in the short run, the individual builds a model of his choice problem, a model which is typically a simplification or a misspecification (or both) of the 'true situation" " (p. 152). However, how do individuals simplify or misspecify the 'true situation' is still a rather unexplored issue.

An important early exception is Thomas Schelling's classic book, The strategy of conflict. Schelling reports John Strachey, the former British Defense Minister, telling him that although he had known that conflict could coexist with common interest, he had thought that the two were inherently separable, and had never considered them as part of an integrated structure (Schelling, 1980, vi). Strachey's words neatly capture an important idea in Schelling's (1980) book: that representing others' strategic motivations may be a source of cognitive difficulty for players when coordination and conflict motives are intertwined in the same game.

For this purpose Schelling introduces a basic and important distinction between "pure motive" and "mixed motive" games. The former are games in which preferences of players are rankcorrelated, as in the prototypical examples of pure coordination games (positive correlation) and conflict games (negative correlation). The latter games present a more complex, non correlated structure of preferences, blending coordination opportunities with antagonistic motivations. The point Schelling makes is that while pure motive games are in general easy to grasp, mixed motive games are puzzling and inherently harder to understand. He strikingly remarks that while our vocabulary is rich of words designating common interest or adversarial relationships, there are no words to designate the relation between players in a mixed motive game ${ }^{1}$ : while we have a rich lexicon for partners or for opponents, how to designate someone who is a partner and an opponent at the same time?

A similar issue has sometimes surfaced in attempts to provide game theoretic prescriptive advice to decision makers. For example, Adam Brandenburger and Barry Nalebuff's (1996) bestseller makes a central argument that managers seldom correctly identify the peculiar mix of competition and cooperation hidden in most business interactions (they feel a revealing need to fill the gap in our dictionaries, coining the hybrid word: co-opetition). Furthermore, it has been repeatedly observed that often bargainers (both in the lab and in real negotiations) tend to miss opportunities to reach agreements on the efficient frontier due to the blinding effects of the "mythical fixed pie bias" (Bazerman, 1983), inducing them to represent the intrinsically mixed-motive bargaining problem as a cognitively simpler constant sum game. Anecdotal evidence from the history of decision making also abounds; for example, Robert McNamara's (1999) recent reappraisal of "missed opportunities" during the Vietnam war provides a rich sample of episodes in which decision makers from both conflicting parties essentially failed to recognize the existence of possible cooperation within conflict and, more generally, recognizes misrepresentations_-"wrong mindsets," in his words—of the nature of the ongoing interaction as a major driver of the evolution of the war.

In this paper, we experimentally address the issue of what makes it difficult for an individual to build a correct mental model of a strategic situation and, following Schelling's intuition, we start by focusing on how different payoff structures present different challenges for the individuals'

1 An associate editor suggested marriage as an example of a typical mixed-motive relationship. 
cognitive ability to represent the game correctly. More specifically, we investigate the difficulty to correctly represent the relations of players' preferences over the game outcomes. For this purpose we introduce the notion of relational complexity of a two-person game representation, and we define it in terms of the structural properties of bi-orders representing the players' payoffs.

Since we want to investigate the extent to which strategic decision making is affected by misrepresentations of the underlying game structure, we proceed in two steps. First, we take a "semantic" stance, and look directly at the cognitive difficulties in representing intertwined order relations which are isomorphic to the preference structures of some classical games. We believe that this may help to disentangle representational factors from other cognitive and behavioral components, and may provide a broader perspective on the difficulties of representing interactive situations. Subsequently, we move to a classical decision making context, looking at how representation difficulties interact with actual decision making-i.e., how some puzzling behaviors may be interpreted in the light of erroneous underlying models of the game.

Our experimental results confirm the appropriateness of our classification for the purpose of understanding individual failures in representing complex relational structures. When facing games of high relational complexity, individuals tend to construct simpler representations, often of a pure-motive type. Thus, although introducing a finer classification, our results confirm that Schelling's insight was essentially correct, i.e., order relations associated to mixed motive games are significantly more difficult to represent than those mirroring pure motive games. We also show that failures in representing order structures of higher complexity are correlated with individual computational capability, as approximated by a measure of short term memory capacity.

In addition, we show that behavior of individuals misrepresenting games is consistent with their erroneous representations. In a way, such individuals play a different game, of a simpler nature. Moreover, since their misrepresentations are most of the time amenable to basic solution concepts, such subjects display behavior consistent with very simple but rational criteria such as dominance or selection of actions supporting payoff-dominant equilibria.

Section 2 of the paper shortly introduces a formal classification of bi-ordered structures, which can be applied to preference relations in two-person games. For this purpose we introduce the property of projectivity. Projectivity and its complement (non-projectivity) well capture, in our view, the degree of entanglement of multiple order relations, as will be better clarified in the next section. Section 3 presents an experiment on the representation of bi-ordered structures and its relation to short term memory capacity. Section 4 describes a behavioral experiment in which the representation task is embedded in actual game playing. We analyze once more representational mistakes and relate them to the interpretation of subjects' strategic behavior. Finally, Section 5 discusses some implications of our results and the relationship with other streams of research in behavioral game theory.

\section{Bi-orders and preference structures}

A game is usually composed of strategies, players (including Nature), and payoffs which determine the players' preferences over the set of outcomes. Sources of cognitive difficulty for individuals may in principle arise from any of these elements alone, or, possibly, from their combination. The complexity of the strategy space is indeed an important source of constraints to players' full rationality in games (chess being the paradigmatic example: e.g., Simon and Schaeffer, 1992), as partisans of bounded rationality have often suggested, and as an abounding experimental evidence by now confirms. 
Much less attention, however, has been paid so far to possible cognitive difficulties arising from the structure of preferences implied by a game. ${ }^{2}$

This diffuse neglect notwithstanding, there is increasing evidence that players can experience serious difficulties in reasoning strategically even in games in which the number of stage-game strategies is very small, as in very simple normal form games (e.g., Stahl and Wilson, 1994; Goeree and Holt, 2001; Devetag et al., 1999; Costa-Gomez et al., 2001). Since in these games strategic complexity cannot arise from the action space, we suggest that one should look at the structure of players' preferences as an important source of difficulty for strategic thinking in such situations. After all, what distinguishes a game situation from an individual decision making task is the need to jointly take into account both one's own and the other players' preferences, and this may indeed result in non trivial complexity even in those cases in which the strategy space is not exceedingly complicated.

In what follows, we restrict our attention to simple, two-person normal form game structures. A peculiar feature of two-person strategic form games is that the outcomes of strategy profiles (i.e., the cells of the bi-matrix) constitute a bi-ordered set, as the preference order of both players is imposed on them. In order to reason strategically on the game, hence, a player must mentally represent two preference orders, her own and the other player's.

In general, bi-orders can have structures of different complexity. A useful typology of biorders, which originated in algebraic linguistics (Marcus, 1967; Schreider, 1975; Mel'cuk, 1987) and which is largely used in the theory of parsing, distinguishes levels of intricacy in the interrelation between two orders on the same set using the properties of monotonicity and projectivity.

Before introducing a few formal definitions, an informal presentation of such properties may be useful.

A bi-order is a pair of order relations (say, $\leftarrow$ and $<$ ) on a set $S$. Let's assume for the sake of simplicity that both relations are linear orders. ${ }^{3} \mathrm{~A}$ bi-order is monotonic if one relation preserves the order of the other (the bi-order is isotonic) or it just reverses it (the bi-order is antitonic). Projectivity can be intuitively expressed by saying that if one writes down the sequence of elements of $S$ according to the < relation, and draws the arrows directly subordinating (i.e., covering) the same elements according to $\leftarrow$, the $\leftarrow$ covering arrows should never cross each other. Finally, a bi-order is non-projective when it is not projective. Non-projectivity can be intuitively expressed by saying that there is no way to arrange the sequence of elements of $S$ according to the $<$ relationship, in such a way that the $\leftarrow$ arrows never cross each other.

Figure 1 shows an example with four elements and two different types of arrows-continuous and dashed-representing the covering relations of $<$ and $\leftarrow$ respectively.

More formally:

Definition 1 (Monotonic projectivity). Let $a_{i}, a_{j} \in S$, and let $\leftarrow$ and $<$ be two linear order relations defined on $S$; a doubly ordered set $S$ is called isotonically projective if:

$$
\text { for } i \neq j \quad a_{i}<a_{j} \quad \text { iff } \quad a_{i} \leftarrow a_{j} .
$$

It is called antitonically projective if:

$$
\text { for } i \neq j \quad a_{i}<a_{j} \quad \text { iff } \quad a_{j} \leftarrow a_{i} .
$$

\footnotetext{
2 Ariel Rubinstein has recently shown some constraints on defining preferences in a simple propositional language: see Rubinstein (2000, Chapter 4).

3 One can generalize definitions to non strict order relations and to the case in which one of the relations is a tree. See for example Schreider (1975).
} 

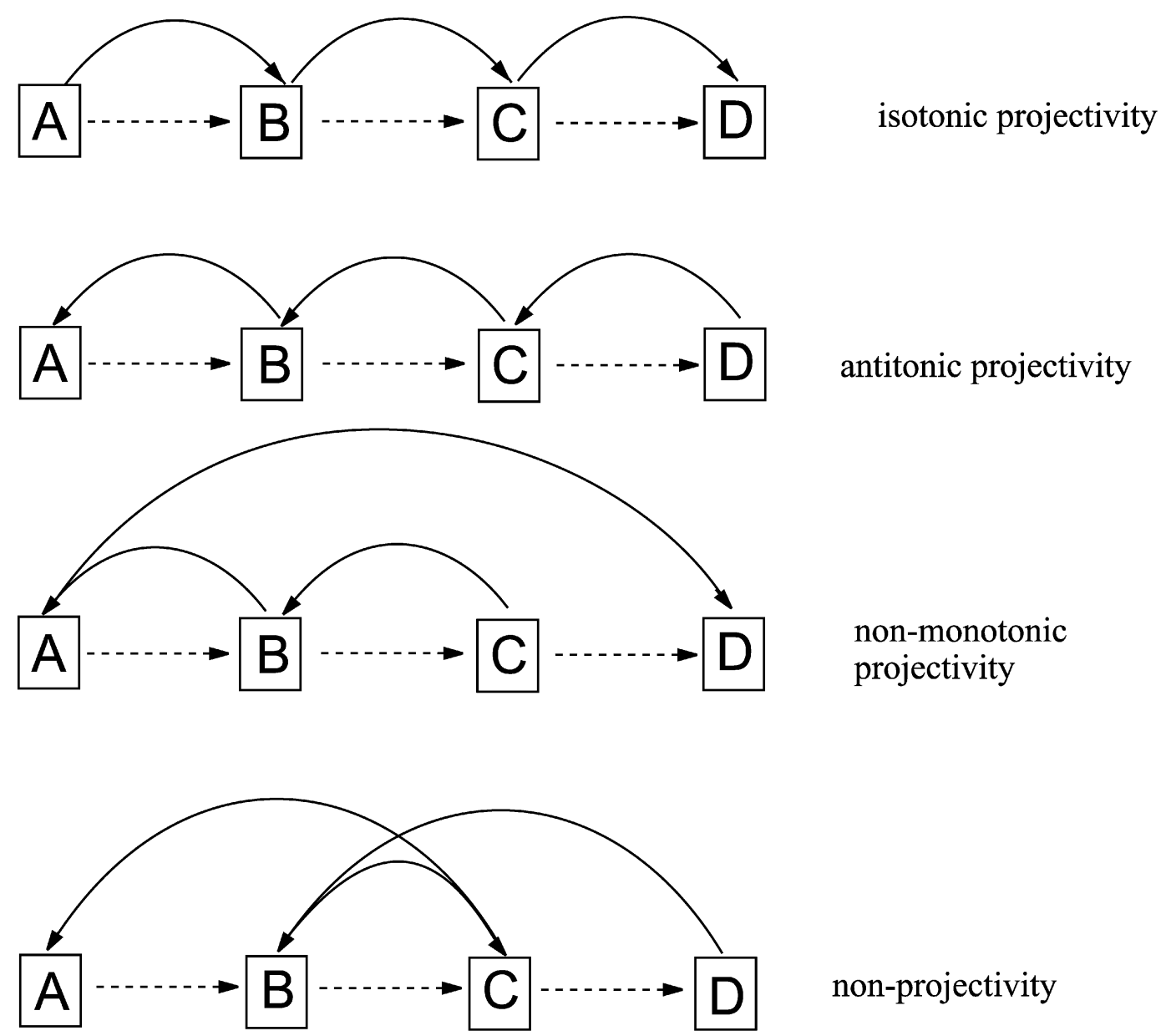

Fig. 1. Four examples of bi-order structures.

It is called monotonically projective if it is isotonically projective or antitonically projective.

Definition 2 (Projectivity). Let $a_{i}, a_{j}, a_{k} \in S$, and let $\leftarrow$ and $<$ be two linear order relations defined on $S$; furthermore, let $\longleftarrow$ be the covering relation of $\leftarrow .{ }^{4}$ A doubly ordered set $S$ is called projective if one and only one of the following conditions holds:

(a) (strict projectivity) for $a_{i} \neq a_{j} \neq a_{k}, a_{i} \longleftarrow a_{j}$ and $\min \left(a_{i}, a_{j}\right)<a_{k}<\max \left(a_{i}, a_{j}\right)$ imply the relation $a_{k} \leftarrow a_{j}$.

(b) (quasi-projectivity) for $a_{i} \neq a_{j} \neq a_{k}, a_{i} \longleftarrow a_{j}$ and $\min \left(a_{i}, a_{j}\right)<a_{k}<\max \left(a_{i}, a_{j}\right)$ imply the relation $a_{i} \leftarrow a_{k}$.

Definition 3 (Non-projectivity). A bi-ordered set is called non-projective if it is neither monotonically projective nor projective.

Since monotonic projectivity is nested into projectivity, one can naturally hypothesize a hierarchy of cognitive difficulty: monotonic projective structures are easier to represent than pro-

\footnotetext{
4 The covering relation for linear orders is usually defined as follows (Davey and Priestley, 1990). Given an ordered set $A$, a linear order relation $\leftarrow$ and $a_{i}, a_{j}, a_{k} \in A, a_{j}$ covers $a_{i}\left(a_{i} \longleftarrow a_{j}\right)$ if $a_{i} \longleftarrow a_{j}$ implies that there are no $a_{k}$ such that: $a_{i} \leftarrow a_{k} \leftarrow a_{i}$.
} 
jective (but non-monotonic) structures, which in turn are easier to represent than non-projective ones. Furthermore, since antitonic projective structures require to reverse one order to obtain the other one, it is reasonable to expect that they may be (slightly) more difficult than isotonic projective structures. Linguistics provides some support to this claim in the domain of language. Language is a system that has multiple order structures simultaneously acting on it: there is the sequential order of words in a phrase, as well as many other layers of syntactical (and semantic) order. For example, to parse a phrase we must be able to recognize and process altogether such order relations. A fairly well explored example is how the linear order of words relates to the dependency order-i.e. the order induced by head-modifier relations in a sentence. A dependency $A-B$ (where $A$ is the governing node of a syntactical tree) is projective iff all the words between $A$ and $B$ are included in the sub-tree of $B$. For example, while "I solved only that same poignant question" is projective, "Solved only that same I poignant question" is non-projective (Schreider, 1975). Empirical analysis (see Marcus, 1967, Chapter 6 for a review) has shown that near $100 \%$ of natural language sentences are projective (with non-projective sentences usually confined to literary usage). More recent, extensive empirical work substantially confirms these results. For example, an analysis of the so-called "Prague dependency treebank," coding a sample of about 30,000 Czech sentences, has found that less than $2 \%$ of the sentences are non-projective (Schwartz, 1998). The importance of projectivity can be understood on the ground that such property allows to introduce a proper bracketing structure into the sentence: in other words, it allows to properly decompose the sentence itself into constituents. This in turn allows to manage complex sentences in the presence of working memory constraints (the interactions between working memory constraints, complex nested sentences, and understanding performance are analyzed in Just and Carpenter, 1992).

Two-person games are bi-ordered structures: our hypothesis is that the cognitive difficulty in representing a game should depend, among other things, on the specific structure of preferences. Pure motive games are monotonically projective structures-in which the two preference relations perfectly coincide - thus they are the easiest to represent; mixed motive games can be of two types: projective ones (like for example "chicken games") or non-projective ones (like for example PDs). The latter should be harder to represent, and therefore understand, than the former.

\section{Representing bi-orders: experiment 1}

\subsection{Description of the experiment and discussion of results}

Our central claim is that there are cognitive constraints in jointly representing multiple order relationships. These constraints seem not specific of game playing only, as the example of language suggests. Thus, we expect them to emerge in more general representational tasks. In order to test our hypothesis, we designed a simple experiment in which subjects were provided with a set of objects that could be ordered by two types of order relations and had to select a subset of them that satisfied such order relations. In semantic terms, the task consists in representing a state of affairs (a "world") that satisfies a formula built up with two order relations. In particular, we tested the hypothesis that different bi-orders induce different levels of representational difficulty. As the reader will remind, we hypothesize the following order of difficulty: non-projective $\triangleright$ projective (but non-monotonic) $\triangleright$ antitonically projective $\triangleright$ isotonically projective, with $\triangleright$ indicating the "more difficult than" relation. 


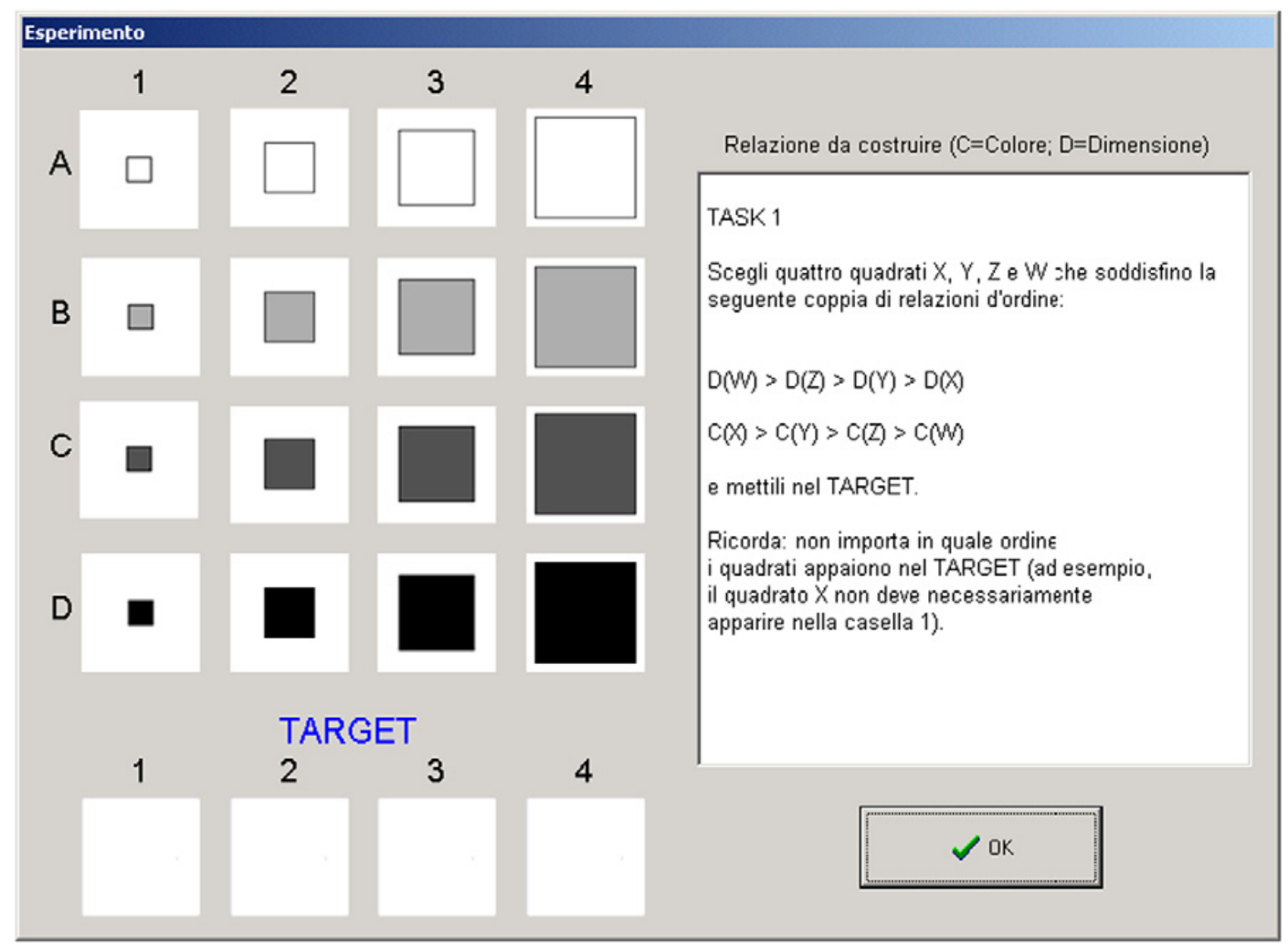

Fig. 2. A sample of the graphical interface used in experiment 1.

The elements our experimental subjects had to deal with were squares which differed along the two features of SIZE and COLOR (actually, shades of gray). Squares are very familiar objects, and size and color are equally familiar order relations, henceforth we expected that no peculiar difficulties could arise in understanding the task. A set of 16 squares was shown to subjects, and their task was to select, out of this set, four squares which would satisfy simultaneously two order relations (size and color) given to them. The experiment was computerized, of the "drag and drop" type (Figure 2 reports a sample of the graphical interface used). The upper part of the screen reported the 16 squares from which subjects had to select their "building blocks." Four empty cells in the bottom part of the screen were the TARGET to be filled in with squares taken from the upper part so as to satisfy the formula. Instructions explained the meaning of order relations and provided examples. ${ }^{5}$

Instructions also stressed the fact that the particular position of the four squares in the TARGET area of the screen did not matter, as long as the four squares satisfied the two order relations given. In order to perform the task, subjects had simply to click with the mouse on one of the squares in the table and "drag" it into one of the cells in the TARGET.

Subjects were presented with the four pairs of order relations shown in Fig. 1, which are orderisomorphic to the payoff structures of the four two-person strategic games shown in Tables 1-4.

In fact, the reader can easily check that the structure of payoffs in the coordination game with Pareto-ranked equilibria is isotonically projective. The pure conflict game corresponds to the case of antitonic projectivity. The projective case is drawn using the chicken game as a template, while the non-projective case is modelled after a Prisoner's Dilemma.

\footnotetext{
5 All the instructions of the experiments reported in this article can be downloaded from http://www.unipg.it/ devetag/ Instructions_GEB.htm.
} 
Table 1

A coordination game (isotonic bi-order)

\begin{tabular}{|c|c|c|}
\hline & $x$ & $y$ \\
\hline$x$ & 3,3 & 2,2 \\
$y$ & 1,1 & 4,4 \\
\hline
\end{tabular}

Table 3

A game of chicken (projective bi-order)

\begin{tabular}{|c|c|c|}
\hline & $x$ & $y$ \\
\hline$x$ & 1,1 & 4,2 \\
$y$ & 2,4 & 3,3 \\
\hline
\end{tabular}

Table 2

A game of conflict (antitonic bi-order)

\begin{tabular}{|c|c|c|}
\hline & $x$ & $y$ \\
\hline$x$ & 1,4 & 3,2 \\
$y$ & 4,1 & 2,3 \\
\hline
\end{tabular}

Table 4

A prisoner's dilemma (non-projective bi-order)

\begin{tabular}{|c|c|c|}
\hline & $x$ & $y$ \\
\hline$x$ & 2,2 & 4,1 \\
$y$ & 1,4 & 3,3 \\
\hline
\end{tabular}

Thus, the following four pairs of order relations were presented to subjects:

- isotonic projectivity (monotonic)

$S(W)>S(Z)>S(Y)>S(X)$,

$C(W) \rightarrow C(Z) \rightarrow C(Y) \rightarrow C(X)$

- antitonic projectivity (monotonic)

$S(W)>S(Z)>S(Y)>S(X)$,

$C(X) \rightarrow C(Y) \rightarrow C(Z) \rightarrow C(W)$

- projectivity (non-monotonic)

$S(X)>S(Y)>S(Z)>S(W)$,

$C(Z) \rightarrow C(Y) \rightarrow C(X) \rightarrow C(W)$

- non-projectivity

$$
\begin{aligned}
& S(X)>S(Y)>S(Z)>S(W), \\
& C(W) \rightarrow C(Y) \rightarrow C(Z) \rightarrow C(X) .
\end{aligned}
$$

$S$ denotes SIZE and $C$ denotes COLOR. The four squares are labeled $X, Y, Z$, and $W$.

A first experimental session was conducted at the University of Venice, and it involved a pool of 30 subjects who were students enrolled in a Master in Business Administration. The subjects had a monetary incentive to give correct responses in the experiment, as they were paid a fixed fee for their participation, plus an amount of 3 euros for each correct answer. The pool was divided into two sub-groups in which the order of presentation of the four bi-orders was varied, to control for learning effects.

The experiment was subsequently replicated with identical conditions at the Computable and Experimental Economics Lab of the University of Trento, using a pool of 40 undergraduate students recruited by posting ads at the various department buildings. Table 5 reports the numbers and relative frequencies of correct responses per task in the Venice and Trento pools respectively, distinguishing between the two sub-groups.

Differences in the number of correct answers between the two sub-groups in each of the four tasks are not statistically significant in both the Venice and Trento pool $(p \geqslant .40$, Fisher exact test): therefore we can refer to the pooled data in the last column of the table. The first thing to notice by looking at aggregate results is that, notwithstanding the relatively better performance of the Trento pool in each task, the observed frequencies of correct answers in both pools suggest an order of difficulty that exactly mirrors our hypothesis: relations which are monotonically 
Table 5

Experiment 1: numbers and relative frequencies of correct answers in the four tasks in the Venice pool (upper part) and in the Trento pool (lower part). The second column reports the order with which the tasks were presented to subjects in the two different sub-groups

\begin{tabular}{|c|c|c|c|c|c|c|c|}
\hline \multirow[t]{2}{*}{ Bi-order } & \multicolumn{3}{|c|}{ group $1(N=14)$} & \multicolumn{3}{|c|}{ group $2(N=16)$} & \multirow{2}{*}{$\begin{array}{l}\text { Tot. } N=30 \\
\text { freq. }\end{array}$} \\
\hline & sequence & number & freq. & sequence & number & freq. & \\
\hline Isot. proj. & 2 & 13 & .93 & 3 & 15 & .94 & .93 \\
\hline Antit. proj. & 1 & 9 & .64 & 4 & 12 & .75 & .70 \\
\hline Proj. & 4 & 7 & .50 & 2 & 10 & .62 & .57 \\
\hline \multirow[t]{2}{*}{ Non-proj. } & 3 & 6 & .43 & 1 & 6 & .37 & .40 \\
\hline & \multicolumn{3}{|c|}{ group $1(N=20)$} & \multicolumn{3}{|c|}{ group $2(N=20)$} & Tot. $N=40$ \\
\hline Isot. proj. & 2 & 19 & .95 & 3 & 19 & .95 & .95 \\
\hline Antit. proj. & 1 & 15 & .75 & 4 & 18 & .90 & .82 \\
\hline Proj. & 4 & 15 & .75 & 2 & 14 & .70 & .72 \\
\hline Non-proj. & 3 & 10 & .50 & 1 & 9 & .45 & .47 \\
\hline
\end{tabular}

projective are relatively easy to construct, with the isotonic one easier than the antitonic. On the contrary, non-monotonic projective and non-projective bi-orders seem relatively more difficult. No statistical differences were found between the observed frequencies in the two experiments ( $p$-values range from .17 to .76, Mann-Whitney $\mathrm{U}$ test), therefore from now on we refer to the pooled data.

Clearly, aggregate analysis alone is not sufficiently informative in this experiment, as the single observations (performance in each task) are not independent. Hence, we performed nonparametric tests on the individual strings of successes (1) and failures (0) in the four tasks to test against the null hypothesis that successes and failures were randomly distributed.

A Cochran test performed on the four related samples allows to reject the null hypothesis that the correct answers in the tasks are equally distributed at the $1 \%$ significance level. ${ }^{6}$ We can hence reject the null hypothesis that the four tasks presented an identical level of difficulty for our subjects.

23 subjects made no mistakes in any of the four tasks, while 2 subjects made the highest possible number (4) of mistakes. Disregarding these 25 subjects' performances as noninformative, out of the remaining 45 subjects, 35 (78\%) behaved in accordance with our conjecture, i.e., they made mistakes in a way that did not violate our hypothesized hierarchy of difficulty. More specifically, 17 subjects made a mistake only in the non-projective task, 9 constructed both monotonic bi-orders correctly but made mistakes in the projective and non-projective tasks, and 9 correctly constructed only the isotonic case. Excluding the extreme cases of everything right and everything wrong, there are fourteen possible strings of 1's and 0's of length 4, of which the only three that are strictly consistent with our conjecture-assuming the sequence isotonicantitonic-projective-nonprojective-are the following: 1-1-1-0, 1-1-0-0-, 1-0-0-0. If all strings were equiprobable, we should expect to find approximately $3 / 14 \times 45=9.2$ strings that confirm our hypothesis, instead of the 35 that appeared in our data. The difference between the observed and the theoretical distribution is statistically significant at the $p=.001$ level by a Chi-square test.

We subsequently made pairwise comparisons by applying a McNemar test. All differences between pairs are statistically significant (isotonic-antitonic bi-order: $p=.002$; isotonic-non-

\footnotetext{
${ }^{6}$ Cochran's $Q=52.796, p=.000$.
} 
projective bi-order: $p=.000$; isotonic-projective bi-order: $p=.000$; antitonic-nonprojective bi-order: $p=.022$; nonprojective-projective bi-order: $p=.003$; McNemar test) except the difference between the antitonic and the projective bi-order $(p=.096)$, which is only weakly significant.

Hence, the data strongly confirm our hypothesis in all but the antitonic-projective pair, although also in this last case observed frequencies of mistakes are as expected.

Additional insight can be gained by conducting an analysis of the most common types of errors that subjects made. While mistakes in the "projective" task show a relatively high variance, mistakes in the "non-projective" task show a rather revealing pattern. In fact, of the thirty-nine subjects who did not answer correctly in this task, twenty (51.3\%) constructed an antitonic biorder, while fifteen $(38.5 \%)$ constructed an isotonic bi-order.

Thus, as we hypothesized, individuals, out of a non-projective pair of relations, tend to simplify their representations by perceiving and extracting monotonic bi-orders.

\subsection{Short-term memory capacity and representation}

Why should some bi-order structures be harder to represent than others? Research in the psychology of mental models (Johnson-Laird, 1983) has repeatedly-although not conclusivelysuggested that short-term memory constraints may hinder the individual ability to edit a complete, accurate mental representation of a given task-environment. Since the pioneering work of George Miller (1956), it is well known that individuals can hold only a limited amount of information active in their short-term memory, which is a basic bottleneck in human information processing. Thus, complex structures may overload individual short-term memory capacity, causing incomplete, over-simplified and often mistaken representations of these structures. The load on short-term memory capacity, however, may be reduced by the ability to compress information or decompose it into smaller components.

Clearly, bi-ordered structures differ in the way information can be compressed or decomposed. For example, isotone bi-orders can be simply processed as a single order, while antitone ones can be easily obtained by reversing a single order. The case of projective and non-projective bi-orders is less trivial. However, projective structures have the property of naturally generating a proper decomposition into a tree of constituents. To see this, it suffices to bracket each pair of elements related by the covering relation $\longleftarrow$ of $\leftarrow$. For example, exploiting the usual order of parentheses and starting from the least element of the chain ordered by $\longleftarrow$, one obtains the bracketing shown in Fig. 3 in the case of a projective bi-order:

This bracketing is "proper," meaning that parentheses are nested.

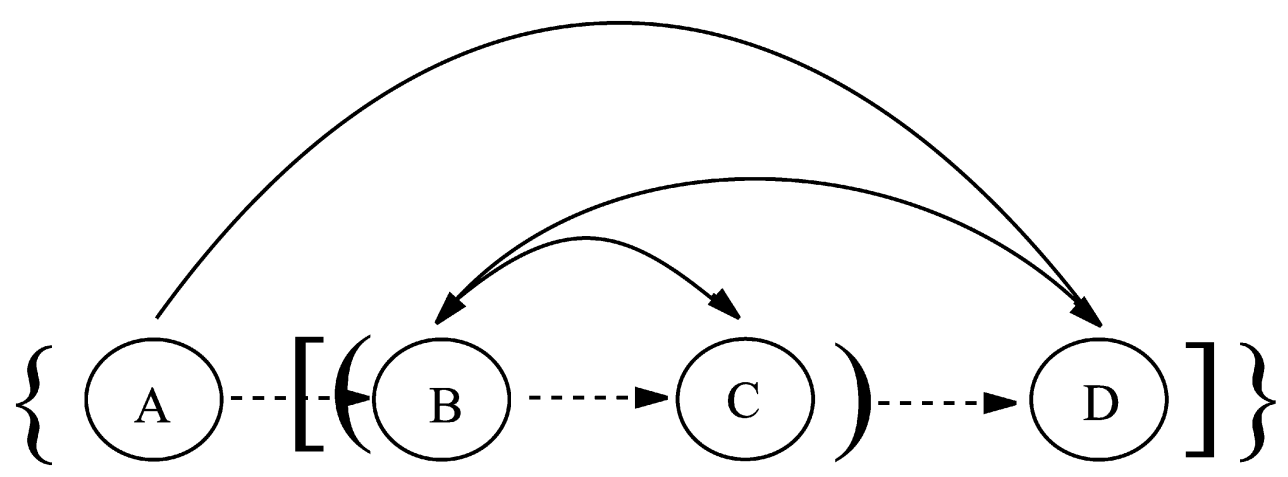

Fig. 3. Bracketing for a projective bi-order. 


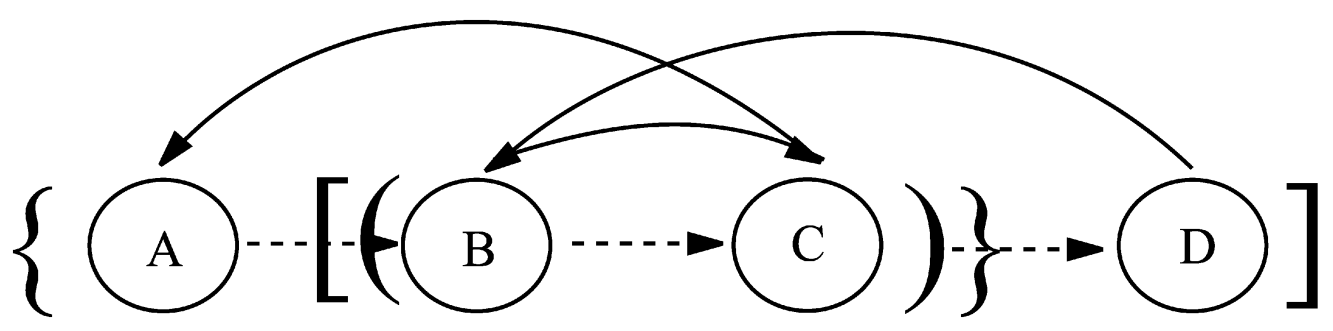

Fig. 4. Bracketing for a non-projective bi-order.

Projectivity implies that a proper bracketing always arises. This follows naturally from the property that the $\longleftarrow$ arrows do not intersect in projective bi-orders.

On the contrary, it is easy to see that non-projective structures fail to generate such decomposition, as it is shown in Fig. 4.

Consequently, there are good reasons to hypothesize that the short-term memory load of editing the representation of a projective bi-order is significantly lowered by the possibility of decomposing it into a tree of constituents (which even a simple stack memory device could easily manage: see Hopcroft and Ullman, 1979). Non-projective bi-orders on the contrary do not present such a decomposability property, and thus force one to consider all elements and their order relations simultaneously.

It has been shown that individuals differ in their short-term memory capacity (Miller, 1956; Baddeley, 1990). Hence, if short-term memory capacity limitations are a source of difficulty in representing bi-orders, one should expect the performance of individuals in our experiment to be correlated with their memory capacity.

In order to test this hypothesis, we conducted a standard Wechsler digit span test for shortterm memory capacity (e.g., Walsh and Betz, 1990; see also Devetag and Warglien, 2003 for a related experiment) on 38 of the 40 subjects of the Trento pool in a separate experimental session. This simple test consists in asking subjects to repeat a series of digits which are to be read by the experimenter at the rate of one digit per second. The test is conducted sequentially on two independent sets of digit series of increasing length. For each set, the test stops when the participant fails to correctly repeat a given series. The subject's 'score' in each set is given by the length of the last series that was repeated correctly (so, for example, if a subject fails to correctly repeat a series of length 6 , her score will be equal to 5). The subject's final score is then given by the higher among the two scores that were achieved in the sets. Although the score needs not directly reflect the number of 'short-term memory slots' available to an individual, it is generally assumed that higher scores correspond to a higher short-term memory capacity.

We computed the correlation between subjects' score in the memory test and the total number of correct responses in the representation experiment. The Spearman rho coefficient equals .310, and the Kendall tau rank-correlation coefficient equals .362 $(p<.05$, one-tailed for both coefficients). Hence, both tests support our hypothesis of a significant correlation between individual short-term memory capacity and performance in the experiment.

Table 6 reports the mean STM score of subjects who were successful and of those who were unsuccessful in each of the four tasks. A $t$-test performed on average scores in the two samples reveals statistically significant differences in the case of the conflict and chicken games, while the differences in the coordination game and the PD are not significant. A plausible explanation for these results, besides the small data sample in the coordination case, is that differences in short term memory bounds are likely to emerge more strongly in tasks of intermediate difficulty. In fact, if the task is very easy most people can solve it no matter how low their memory capacity 
Table 6

Average STM score of subjects who err and of subjects who don't err in each task. The first number between parentheses reports the corresponding standard deviations, and the second number reports the number of subjects in each category. The last column reports the $p$-value of the $t$-test for equality of means

\begin{tabular}{llll}
\hline task & unsuccessful subjects & successful subjects & $p$-value \\
\hline coordination (isotonic) & $5.3(1.5 ; 3)$ & $5.9(.9 ; 35)$ & .563 \\
conflict (antitonic) & $5.2(.44 ; 5)$ & $6(1 ; 33)$ & .011 \\
chicken (projective) & $5.4(.52 ; 9)$ & $6.03(1.05 ; 29)$ & .033 \\
PD (non-projective) & $5.8(.78 ; 19)$ & $6(1.15 ; 19)$ & .516 \\
\hline
\end{tabular}

is, and if the task is very difficult the workload is such that even people with high scores can make mistakes, as our data on the coordination game and the PD show.

To strengthen this explanation, look at the correlation between memory scores and the number of correct responses in the coordination and conflict games only (Kendall's tau-b $=.275$, $p=.036$, and Spearman's rho $=.297, p=.035)$, or between memory scores and numbers of correct responses in all tasks except the PD (Kendall's tau-b $=.297, p=.025$, and Spearman's rho $=.321, p=.025)$. The values indicate that a considerable portion of the correlation found is preserved by restricting its calculation to performance in the monotonic and projective tasks only.

\section{Experiment 2: representation of games and links with behavior}

\subsection{Experimental design and implementation}

Having verified the cognitive difficulty of representing different classes of bi-orders, our second experiment is aimed at assessing how these difficulties affect game playing. For this purpose, we designed an experiment in which subjects had to construct a representation of the four games depicted in Tables 1-4, and subsequently choose a strategy in each game. The experiment was divided in two parts. At the beginning of the experiment, subjects were told that in the second part they would participate in four interactive decision making tasks, and in each task they would be paired with a randomly selected opponent. In each decision making task each player could choose between two available moves. The four resulting combinations of choices generated four different scenarios (called $A, B, C$ and $D$ ), each implying different payoffs for the two players. The possible payoffs in each game could range from 1 to 4 experimental points for each player, and would be represented by squares differing in color and size. A player's payoff depended on color (the darker the square, the higher the payoff), while the other player's payoff depended in the same way on the square size. In the first part of the experiment subjects would have to visually represent the four games according to pairs of order relations like the one in experiment 1 . This was done by selecting, among a set of 16 squares, the four that satisfied the relations given; hence, the first part of the experiment was essentially equivalent to experiment 1 , with the difference that subjects knew that they were representing interactive decision making tasks that would be the object of the second part of the experiment, and that the color and size of the squares represented their and their opponents' payoffs in the games. Since our interest is in bi-orders, also in this case we kept a geometric, and hence purely ordinal, representation of payoffs avoiding their direct translation into numbers. For the same reason, we avoided imposing further representational structure by using devices such as game matrices, leaving instead the simple linear display of geometric figures used in experiment 1.

The instructions stated that the representational task was strictly individual and that earnings in that task were solely a function of the number of games correctly represented, whereas earn- 
ings in the second part were contingent on one's own and one's opponent choices in the games. In order to emphasize the effects of working memory constraints we introduced a time limit of 120 seconds to complete each representation, and the four games appeared to each subject in a random order to control for learning effects.

In the second part of the experiment, each subject's computer terminal displayed the four representations that she had constructed in the first part, one at a time, and the subject had to choose a move between the two available. Hence, subjects had to play the games according to the representation that they themselves had constructed, knowing they would be randomly paired with a different opponent in each game, and all this information was common knowledge. The instructions also specified that in the case a subject had not been able to complete a representation within the time limit, the representation would be constructed arbitrarily by the computer program. Furthermore, the instructions stated that the payoffs in the games were calculated according to the moves chosen by the two players on the basis of the correct representation. In this way, a further incentive was introduced to represent the game correctly. At the beginning of each game, players were randomly assigned to either COLOR or SIZE (corresponding to the usual ROW and COLUMN roles in game matrices), and the two available moves were labeled with the two symbols of spades and clubs. Subjects did not receive any form of feedback at any time regarding their performance and/or their opponents' decisions. Figure 5 reports a sample of the

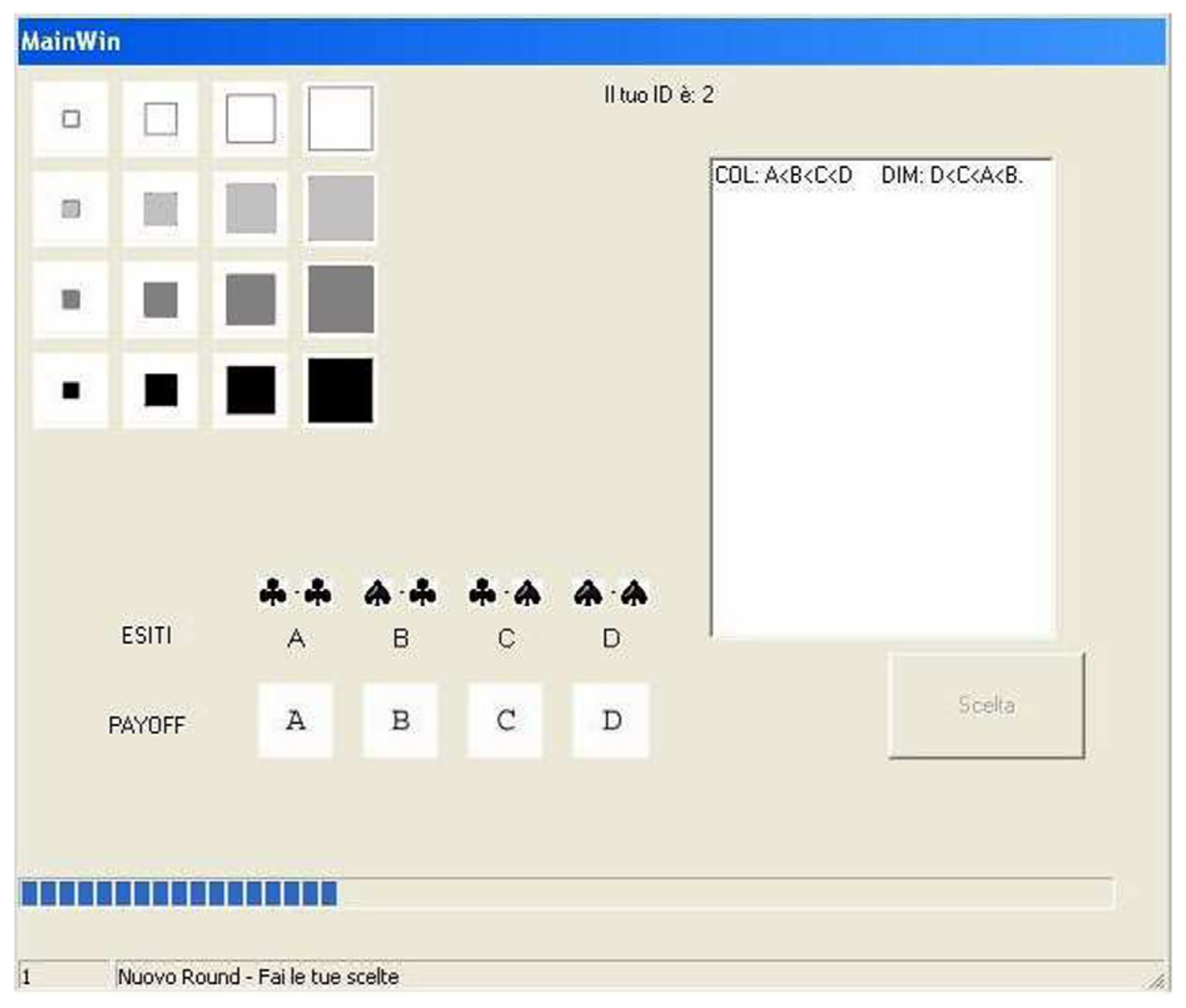

Fig. 5. A sample of the graphical interface used in the first part of experiment 2. 
graphical interface used in the first part of the experiment. ${ }^{7}$

The experiment was run at the Computable and Experimental Economics Lab of the University of Trento in three separate sessions and it involved a total of 68 subjects, who were all undergraduate students who had never participated in similar experiments before. Instructions were distributed at read aloud at the beginning of the experiment. The overall time limit for the first part was set equal to eight minutes, whereas no time constraint was imposed in the second part. There was a show up fee of 3 euros plus anything subjects could earn in the experiment. Average overall earnings were equal to 14.8 euros; the experiment lasted sixty minutes on average, including the instruction time.

\subsection{Results}

Table 7 reports the frequencies of correct responses in each representation task. The observed frequencies in the different games mirror very closely those in experiment 1 . The Cochran test performed on the four samples is highly significant $(p=.000)$. Likewise, pairwise differences measured by a McNemar test are all statistically significant (coordination-chicken game: $p=.002$; coordination-PD: $p=.000$; conflict-chicken game: $p=.029$; conflict-PD: $p=.000$; chicken-PD: $p=.032$ ), although the difference between the coordination and the conflict games is only weakly so $(p=.10)$.

23 subjects made no mistakes in any of the four tasks, and 4 subjects made four mistakes. Hence, there is a total of 41 subjects who made from 1 to 3 mistakes, and whose performance is therefore informative. A qualitative analysis of the individuals strings of successes (1) and failures (0) reveals that 26 out of 41 strings (63\%) are strictly consistent with our hypothesized hierarchy of difficulty, against the $22 \%$ that would be expected in case of randomly distributed errors (the difference is significant at the .001 level by a Chi-square test). ${ }^{8}$

We then move to an in-depth analysis of the mistaken representations, which we restrict to the chicken game and to the Prisoner's Dilemma, because the number of errors in these two tasks is sufficiently high.

Our conjecture is that the erroneously reconstructed matrices should diverge from the correct ones in terms of their relational structure, and in particular that they should reflect the difficulty of representing complex bi-orders.

Table 7

Experiment 2: numbers and relative frequencies of correct answers in the representation task

\begin{tabular}{ll}
\hline task & correct answers \\
\hline coordination (isotonic) & $59(.87)$ \\
conflict (antitonic) & $53(.78)$ \\
chicken (projective) & $45(.66)$ \\
PD (non-projective) & $35(.51)$ \\
\hline
\end{tabular}

\footnotetext{
7 A figure displaying the graphical interface used in the second part can be downloaded from http://www.unipg.it/ $\sim$ devetag/Instructions_GEB.htm.

8 This analysis, as well as the analogous analysis in experiment 1, would give the same results if we included the strings of subjects who did everything right and those of the subjects who made four mistakes. In fact, although these cases are not informative, they are not, as such, in contrast with our hypothesized hierarchy of difficulty.
} 
Table 8

Experiment 2: empirical distribution of erroneous representations classified by typology of bi-order (between parentheses: number of subjects that best respond to their representation)

\begin{tabular}{lllll}
\hline & isotone & antitone & projective & non-projective \\
\hline chicken game $(n=17)$ & $6(4)$ & 0 & $9(9)$ & $2(2)$ \\
$\operatorname{PD}(n=23)$ & $5(4)$ & $9(8)$ & $3(3)$ & $6(5)$ \\
\hline
\end{tabular}

Table 8 reports the empirical distribution of mistaken games classified by type of bi-order. The numbers in parentheses report how many subjects chose a best reply given their (mistaken) representation. A useful benchmark is the distribution of possible classes of bi-orders in $2 \times 2$ matrices. It turns out that, out of the $4 !=24$ possible $2 \times 2$ matrices that one can build, only 1 is isotonic, only 1 is antitonic, 14 are projective and 8 are non-projective. In both the chicken and the PD case, the distribution of types of bi-orders generated by our subjects significantly differs from such distribution ( $p=.0003$ for the PD case, and $p=.02$ for the chicken case, according to a Chi-squared test with simulated $p$-value, 1,000,000 replicates). Furthermore, according to the same test, the "erroneous PD" distribution is significantly different from the "erroneous chicken" one $(p=.002)$. In other words, mistakes in the PD and in the chicken game are drawn from different distributions.

Thus, representational errors are not random. In both cases, they show more isotonic/antitonic instances and less projective/non-projective ones than randomly expected $(p<.01$, one-tailed Fisher exact test). At the same time, mistakes seem to reflect the structure of the underlying representation problem: there are more projective than non-projective mistaken representations in the chicken game, and the reverse holds in the case of PD ( $p=.04$, one-tailed Fisher exact test). Moreover, the chicken game is more often associated with isotonic representations than with antitonic ones, while the reverse is true for the PD game $(p=.01$, one-tailed Fisher exact test). Thus, the chicken game is often transformed into a coordination game, while the PD game is often transformed into a pure conflict game, although sometimes it still is transformed into a coordination game.

An important issue is how representations interact with actual choice behavior. As far as subjects that represent the games correctly are concerned, choices in the four games conform to well-established behavioral principles (see Table 9). In the coordination game, subjects predominantly play the strategy that supports the payoff dominant equilibrium, although, especially when the two strategies have the same payoff sum, as in the case of row players, many play the alternative, safer strategy. In the conflict game the distribution of behavior is different from the mixed strategy equilibrium probabilities, due to a large extent to the fact that subjects take into consideration the weight of out-of-equilibrium payoffs, as related experiments have shown (e.g., Goeree and Holt, 2001). In the chicken game, the safer deferential action is more often played than the more risky and aggressive one. Finally, in the PD about 2/3 of players defect, but another third is cooperating.

Looking at the behavior of those who have misrepresented the game is more informative. We have suggested that, in general, when misrepresenting a game subjects tend to generate representations which are structurally simpler than the "correct" one. Often these representations also imply simple solution criteria. As a result, although subjects often construct the wrong game representation, they act quite rationally in the light of such erroneous constructs.

The data reported in Table 8 on the number of subjects who best respond to their erroneous representations seem to strongly support this claim, in that almost all subjects choose rationally 
Table 9

Experiment 2: relative frequencies of choices of actions in each game, disaggregated by row and column role for the non-symmetric games. Only the choices made on the basis of correct representations are reported

\begin{tabular}{llllll}
\hline & & \multicolumn{3}{c}{ Games } \\
\cline { 3 - 6 } & & Coordination & Conflict & Chicken game & PD \\
\hline row & $x$ & .45 & .19 & .36 & .68 \\
& $y$ & .55 & .81 & .64 & .32 \\
col & $x$ & .16 & .48 & n.a. & n.a. \\
& $y$ & .84 & .52 & n.a. & n.a. \\
& & & & &
\end{tabular}

in each category of 'simplified games.' A more detailed analysis of some of these errors and the resulting choices is revealing: for example, 11 out of 17 subjects who have misrepresented the chicken game have reconstructed it as a game in which a single strategy profile yields the maximum payoff for both players-and 9 out of these 11 subjects have played the strategy corresponding to such profile. Of those 11 representations, 8 reconstructed the chicken game as a coordination game with a Pareto-dominant equilibrium-and in 6 out of these 8 representations subjects have played the strategy corresponding to that equilibrium. 5 of the 6 representations that do not have a "common max" profile have a dominant action-and once more 4 out of 5 subjects play according to dominance.

Similarly, 21 out of 23 subjects who have misrepresented the PD game have introduced a dominant strategy in their representation, and have acted almost always (18 out of 21) according to dominance. Notice, however, that the dominant strategy in the erroneous representations of the PD game does not necessarily correspond to defection: 8 players represent "cooperate" as the dominant action (and 6 play according to it). From a different angle: 8 out of 23 subjects who have misrepresented the PD game have played "cooperate" (i.e., have picked the strategy that is labeled as cooperation in the correct game) and 7 of these 8 subjects have done it according to a principle of dominance or Nash equilibrium. If these apparent cooperators are more rational than one might have guessed, "defectors" with erroneous representations are not really defecting in the game they have reconstructed: indeed, all but one of them have built a game in which the equilibrium in dominant strategies for both players is also the social optimum. In summary, no matter whether they cooperate or defect according to the labels that apply in the "true" game, most players that erroneously reconstructed the PD represented it in such a way that the social optimum is also an equilibrium.

\section{Discussion and conclusion}

Our experiments provide support for the view that not all normal form games are the "same," and that structural complexity matters; we suggest that besides the strategy space, relational structure is a further source of cognitive difficulty, providing a finer classification of two-person games. "Pure motive" games (i.e. monotonic payoff structures) are easier to represent, and even in the presence of "mixed motive" games, such simpler structures act as irresistible templates of interaction. We also show that a further classification, involving the property of projectivity, is useful in defining levels of relational complexity.

We also show that the same ranking of representational difficulty applies when bi-orders are explicitly embedded in the presentation of a game. Subjects aware that they are setting the stage for interactive decisions experience the same increasing difficulty as the payoff structure goes 
from isotonicity to non-projectivity. The analysis of the behavior of subjects that misrepresent the games reveals a few relevant features. (1) Subjects tend to simplify representations, constructing models of the game that lower its relational complexity. (2) In doing so, they still anchor to some structural features of the "true" game. For example, in the PD, subjects still generate more non-projective erroneous representations than in the chicken game. Furthermore, other features of the correct game are preserved, although probably as a bi-product of other representational processes; for example, dominance is preserved in most erroneous PDs, whereas multiplicity of equilibria emerges in many erroneous chicken games. Given such simplified representations, actual choice behavior follows simple but quite "rational" decision criteria, such as dominance or selection of actions implementing payoff-dominant equilibria. These results suggest a fresh reinterpretation of behavior in well known experimental games. We have shown that when subjects misrepresent the PD game, they tend to eliminate its social dilemma nature, in a way that both the "cooperation" and "defection" moves actually correspond to strategies that support perceived social optima.

Similarly, behavior in the chicken game can be sometimes interpreted as the result of its subjective reframing as a coordination game with a payoff-dominant equilibrium.

Other well-documented behaviors find a natural interpretation in this framework. For example, a large literature in negotiation research has pointed out that bargainers are often subjects to the "mythical fixed pie bias" (Bazerman, 1983), which causes them to wrongly assume that their interests are diametrically opposed. "The assumption of a fixed pie is rooted in social norms that lead us to interpret most competitive situations as win-lose [...]. Humans tend to generalize from these objective win-lose situations to situations that are not necessarily win-lose" (Bazerman et al., 2001, p. 13). Within our framework, the fixed pie bias is almost literally the transformation of a complex bargaining game into a simpler, antitonic, antagonistic representation. Some further evidence in this sense comes from a recent experiment on complex multi-issue negotiations (Hyder et al., 2000). The authors of the study observe that negotiators rarely achieve a Pareto-optimal solution to a given negotiation problem, and they argue that the reason lies in their incorrect 'default' representation of the situation as a zero-sum game. In fact, representing the game as zero-sum would trigger the almost exclusive use of distributive negotiation tactics (i.e., tactics aimed at achieving unilateral concessions from the other party) at the expense of integrative tactics, which would instead facilitate the achievement of agreements resulting in gains for both parties involved. Hence, the use of specific behavioral strategies conducting to suboptimal agreements seems to derive, according to the authors, by an original failure of players to represent the mutual gain area in the space of solution points.

Incidentally, it should be noted that games that present a more complex structure are also more likely to be affected by superficial cues or attention-directing devices pointing to some simpler interaction structure. Such cues might act as cognitive affordances driving the construction of a simpler representation. For example, Ross and Ward (1996) have observed that behavior in the Prisoner's Dilemma may be directed towards cooperation or defection by manipulating its labeling either as a "Wall Street game" or "Community game." Pillutla and Chen (1999) found similar contextual effects in an experiment on Public Goods games. While of course such effects may also resent of the weight of social norms and stereotypes of appropriate behavior, we submit that the need for cognitive simplification may play an important permissive role in triggering the search for principles that facilitate the construction of a new representation.

In addition, our results point to an important source of heterogeneity in a population of game players, namely differences in the relative ability to correctly represent the structure of strategic interaction. These differences are in parallel with observed differences in the 
depth of iterated thinking in games (e.g., Nagel, 1995; Camerer, 2003, Chapter 5; Camerer et al., 2002). Interestingly enough, both types of heterogeneity appear to be (weakly) correlated with differences in short term memory capacity, a measurable psychological proxy of individual computational capability (Newell and Simon, 1972; Devetag and Warglien, 2003; Kareev, 1992). These observations may also help understand how players transfer behavior from previously experienced games to new ones. Knez and Camerer (2000) show that after playing a common interest coordination game, individuals are more prone to cooperate in a PD. We suggest that the coordination game may act as a template for the cognitive simplification of the subsequent PD. In fact, our taxonomy may help to predict the direction of transfer phenomena from simpler to more complex games.

Furthermore, we submit that monotonic structures may indeed be the prevailing templates of bi-orders available in memory. In a classroom experiment, students asked to provide examples of four arbitrary objects satisfying simultaneously two arbitrary order relations of the kind depicted in Fig. 1 had no difficulties in finding examples for monotonic bi-orders (such as "richer is happier" or "larger towns are less healthy"); but they found it almost impossible to provide examples for non-projective bi-orders. This point reinforces the result that in our experiment subjects unable to provide a solution to the non-projective case resorted to monotonic orderings of the squares. Returning to games, it also suggests that in incomplete information games "friends" and "enemies" may be the most natural player types.

Our results may also provide some complementary cognitive ground to Ariel Rubinstein's (1996) argument on the prevalence of linear order structures in discourse. Rubinstein claims that linear orders have some efficiency properties (in indicating an element out of a set, in being informative about a relation on a set, in minimizing the number of examples necessary to describe a relation) that justify the higher frequency with which these structures appear in natural language. Clearly, one can construct a structure-preserving map from a monotonic bi-order to a linear order, either directly (as in the isotonic case) or with an intermediate step by reversing one of the two order relations (as in the antitonic case). The same cognitive constraints that make monotonic bi-orders easier to represent may underlie the prevalence of linear orders in natural language. Projectivity is a more complex case: no simple way to reduce it to a single linear order can be found. Yet, projectivity can be thought of as a kind of compatibility between order relations, simplifying the task of managing bi-orders in short term memory. The relevance of the projectivity property in natural language suggests that further connections with Rubinstein's argument are worth seeking.

Finally, our results provide a first, albeit partial, answer to what Colin Camerer (2003) has placed among the top ten open research questions in behavioral game theory, namely: "What game do people think they are playing?" (p. 474). Experimental game theory has so far relied on the implicit assumption that the game subjects played was the one provided by the experimenter. Our data suggest that this assumption may be misleading, and that, more generally, individuals may indeed apply optimal decision criteria to a misspecified strategic setting.

\section{Acknowledgments}

Financial support from the Italian Ministry of University and Research (PRIN 2005) and from the Cognitive Science Laboratory of Rovereto is gratefully acknowledged. We thank Yaakov Kareev, Marco LiCalzi, Andreas Ortmann, Burkhard Schipper, Reinhard Selten, seminar participants in Graz, Lyon, Barcelona, Prague and Rome, two anonymous referees and an associate editor for useful comments and suggestions. We thank Marco Tecilla and Ivan Soraperra for the design of the experimental software. The usual disclaimer applies. 


\section{References}

Baddeley, A.D., 1990. Human Memory: Theory and Practice. Erlbaum Associates, Hillsdale, NJ.

Bazerman, M.H., 1983. Negotiator judgment: A critical look at the rationality assumption. Amer. Behav. Sci. 27, 618634.

Bazerman, M.H., Baron, J., Shonk, K., 2001. Enlarging the societal pie-A cognitive perspective. Working paper No. 02-081, Harvard Business School.

Brandenburger, A.M., Nalebuff, J.N., 1996. Co-opetition. Doubleday, New York, NY.

Camerer, C., 2003. Behavioral Game Theory: Experiments on Strategic Interaction. Princeton Univ. Press, Princeton, NJ.

Camerer, C., Ho, T., Chong, J.K., 2002. A cognitive hierarchy theory of one-shot games: Some preliminary results. Working paper, California Institute of Technology.

Costa-Gomez, M., Crawford, V., Broseta, B., 2001. Cognition and behavior in normal-form games: An experimental study. Econometrica 69, 1193-1235.

Davey, B.A., Priestley, H.A., 1990. Introduction to Lattices and Order. Cambridge Univ. Press, Cambridge, UK.

Devetag, G., Warglien, M., 2003. Games and phone numbers: Do short term memory bounds affect strategic behavior? J. Econ. Psych. 24, 189-202.

Devetag, M.G., Legrenzi, P., Warglien, M., 1999. Focusing in naive game playing. In: Schaeken, V., De Vooght, G., Vandierendonck, A., d'Ydewalle, G. (Eds.), Deductive Reasoning and Strategies. Erlbaum Associates, NJ, pp. 287299.

Goeree, J.K., Holt, C.A., 2001. Ten little treasures of game theory and ten intuitive contradictions. Amer. Econ. Rev. 91, 1402-1422.

Hopcroft, J.E., Ullman, J.D., 1979. Introduction to Automata Theory, Languages and Computation. Addison-Wesley, Reading, MA.

Hyder, E.B., Prietula, M.J., Weingart, L.R., 2000. Getting to best: Efficiency versus optimality in negotiation. Cognitive Sci. 24 (2), 169-204.

Johnson-Laird, P.N., 1983. Mental Models. Cambridge Univ. Press, Cambridge, UK.

Just, M.A., Carpenter, O.A., 1992. A capacity theory of comprehension. Psych. Rev. 99, 122-149.

Kareev, Y., 1992. Not that bad after all: Generation of random sequences. J. Exper. Psych. Human Perception Performance 18, 1189-1194.

Knez, M., Camerer, C., 2000. Increasing Cooperation in prisoner's dilemmas by establishing a precedent of efficiency in coordination games. Organ. Behav. Human Dec. Process. 82 (82), 194-216.

Kreps, D., 1990. Game Theory and Economic Modeling. Oxford Univ. Press, Oxford.

Marcus, S., 1967. Algebraic Linguistics: Analytical Models. Academic Press, NY.

McNamara, R., 1999. Argument Without an End. Public Affairs, NY.

Mel'cuk, I.A., 1987. Dependency Syntax: Theory and Practice. State Univ. of New York Press, Albany, NY.

Miller, G., 1956. The magical number seven, plus or minus two. Some limits of our capacity for processing information. Psych. Rev. 63, 81-97.

Nagel, R., 1995. Experimental results on interactive competitive guessing. Amer. Econ. Rev. 85, 1313-1326.

Newell, A., Simon, H.A., 1972. Human Problem Solving. Prentice Hall, Englewood Cliffs, NJ.

Pillutla, M., Chen, X.P., 1999. Social norms and cooperation in social dilemmas: The effects of context and feedback. Organ. Behav. Human Dec. Process. 78 (2), 81-103.

Ross, L., Ward, A., 1996. Naive realism: Implications for social conflict and misunderstanding. In: Brown, T., Reed, E., Turiel, E. (Eds.), Values and Knowledge. Erlbaum Associates, Hillsdale, NJ, pp. 103-135.

Rubinstein, A., 1996. Why are certain properties of binary relations relatively more common in natural language? Econometrica 64, 343-356.

Rubinstein, A., 2000. Economics and Language. Cambridge Univ. Press, Cambridge, UK.

Schelling, T., 1980. The Strategy of Conflict, second ed. Harvard Univ. Press, Cambridge, MA.

Schreider, J.A., 1975. Equality, Resemblance and Order. MIR Publishers, Moscow, USSR.

Schwartz, O., 1998. Structured language model parsing. In: Core Natural Language Processing Technology Applicable to Multiple Languages: Final Report, Structured Language Model Parsing. CLSP, Johns Hopkins University.

Simon, H.A., Schaeffer, J., 1992. The game of chess. In: Aumann, R.J., Hart, S. (Eds.), In: Handbook of Game Theory with Economic Applications, vol. 1. Elsevier Science, The Netherlands, pp. 1-17.

Stahl, D.O., Wilson, P.W., 1994. Experimental evidence on players' models of other players. J. Econ. Behav. Organ. 25, 309-327.

Walsh, W.B., Betz, N.E., 1990. Tests and Assessment. Prentice Hall, Englewood Cliffs, NJ. 